SRC TR $85-52$

A Local Version of The Two-Circles Theorem

C. A. Berenstein

and

R. Gay 


\section{A local version of the two-circles theorem}

by

C. A. Berenstein and R. Gay

Abstract: A necessary and sufficient condition is given so that in a domain $\Omega$ there are no functions whose average over all balls contained in $\Omega$ of radii $r_{1}, r_{2}$ vanish except the zero function.

Key words: Convolution equations, two-circles theorem, Radon transform.

A.M.S. subject classification: 30E99, 31B99, 42A75 

A local version of the two circles theorem

by

Carlos A. Berenstein*

Mathematics Department

University of Maryland

College Park, MD 20742 USA

and

\author{
Roger Gay \\ Universite de Bordeaux I. \\ U.E.R. de Mathematiques et Informatique \\ Unite Associee au C.N.R.S. N 040226 \\ 351 , Cours de la Liberation \\ 334505, Talence Cedex, France
}

*Partially supported by NSF grant DMS-8401356 and by NSF grant OJR 85-OV-108 through the systems Research center of the University of Maryland. 


\section{Introduction. -}

One of the oldest questions in integral geometry has been that of recovering a function $f$ in $R^{n}$ from the knowledge of its average over balls. It is easy to see that unless f decays sufficiently fast at infinity the average over all balls of a fixed radius could vanish without $f$ being identically zero. It is not always possible to assume such decay but a very elegant result of zalcman [20] and, independently, Brown-Schreiber-Taylor [10], describes explicitly a countable set $E_{n}$ such that averages over all balls of radii $r_{1}, r_{2}$ suffice as long as $r_{1} / r_{2} \neq E_{n}$. This-"two circles" theorem can be described as saying that the map

$$
\begin{gathered}
C\left(R^{n}\right)+C\left(R^{n}\right) \oplus C\left(R^{n}\right) \\
E \rightarrow\left(\int_{B(x, r, f} f(y) d y, \int_{B\left(x, r_{2}\right)} f(y) d y\right)
\end{gathered}
$$

is injective if and only if $r_{1} / r_{2} \notin E_{n}$. $\left(B(x, x)=\left\{y R^{n}:|x-y|<x\right\}\right)$. Under slightly stronger conditions on the quotient $r_{1} / r_{2}$ this map has also a continuous and explicit inverse [8]. This result and other variants of the so-called Pompeiu problem have been generalized to symetric spaces (see the surveys [20], [1] for positive results and their limitations] In practical situations of a tomographic nature one is limited to balls that fit into a fixed region $\Omega$. One could take smaller and smaller balls when approaching the boundary $\partial \Omega$ of $\Omega$, this is roughly the situation when we consider the case 
$\Omega=$ unit ball of $R^{n}$ as the hyperbolic space, but it is clear that it might be hard to accomplish if we are dealing with physical devices whose size cannot be made infinitesimally small or cannot even be changed at will. It is this kind of problem that we call a local version of the two-circles theorem. The main difference with the above mentioned results is that we do not have any longer the whole group of Euclidean motions at our disposal which was the crucial ingredient lying behind the two circles theorem and its generalizations. The inversion formula of [8] would allow us to reconstruct $f$ away from $a \Omega$ but gives no indication of whether we could change the values of $f$ in a collar-like region near $\partial \Omega$ without affecting its average. There is some recent work on systems of convolution equations in convex domains which deals with this type of question [4] but the hypotheses required are far too restrictive to be satisfied by our simple looking problems. Nevertheless, using a combination of ideas from classical harmonic analysis and results of cormackQuinto on the Radon transform on spheres [12] we are able to prove the following.

Theorem. - Let $r_{1}, r_{2}$ be positive numbers; $r_{1} / r_{2} \notin E_{n}$ ' $\Omega$ an open subset of $\mathbf{R}^{\mathbf{n}}$ such that every point lies in an open ball contained in $\Omega$ of radius strictly larger than $r_{1}+r_{2}$. If $f \in C(\Omega)$ satisfies

$$
\int_{B\left(x, r_{j}\right)} f(y) d y=0 \text { for every } \bar{B}\left(x, r_{j}\right) \subseteq \Omega, j=1,2
$$


then $f \equiv 0$. Furthermore, this statment does not hold if $\Omega$

fails the above geometrical restriction.

The method of proof allows us to generalize this theorem greatly, providing in particular new local mean-value theorems for harmonic functions.

We will like to express our appreciation to professor $L$. zalcman who called our attention to these problems.

The second author wishes to thank the Mathematics Department of the University of Maryland for its hospitality while this work was carried out.

2. Preliminaries. -
we will follow the standard notation for distributions found in $[14]$. We denote $B(x, r)=\left\{y \in R^{n}:|x-y|<r\right\}(x>0), \bar{B}(x, x)$ its closure and $x_{r}$ the characteristic function of $B(0, r)$. Iet $\Omega$ be an open set in $R^{n}, \Omega_{r}=\{x \in \Omega: d(x, \Omega c)<r\}$. For a locallyintegrable function $f$ in an open set $\Omega$ the average

$$
A_{r}(f, x)=\frac{1}{\omega_{n} r^{n}} \int_{B(x, r)} f(y) d y
$$

is defined for $x \in \Omega_{r}$. Here $\omega_{n}$ is the volume of $B(0,1)$. If we let $\mu_{r}=x_{r} / \omega_{n} r^{n}$, we can interpret this average as a convolution and hence it makes sense to define it for $f \in D^{\prime}(\Omega)$ giving a distribution $A_{x}(f)$ in $D^{\prime}\left(\Omega_{r}\right)$, namely $A_{r}(f)=f * \mu_{r}$ Therefore, for uniqueness questions, if the averages of $f$ are zero, by restriction ourselves to $\Omega_{\varepsilon} \varepsilon^{\prime} 0$ small, we can assume $f \in c^{\infty}$. Henceforth, all distributions with vanishing averages will be assumed to be co functions in $\Omega$. 
For $r>0$, we denote by $\sigma_{r}$ the distributions defining the spherical average

$$
\lambda_{r}(f, x)=\int_{S} n-1 f(x+r y) d \sigma(y)=\left(\sigma_{r}^{\star} f\right)(x)
$$

do is the normalized Lebesgue measure on $\mathrm{s}^{\mathrm{n}-1}$.

For $T \in E^{\prime}$ the Fourier transform

$$
\hat{T}(\zeta)=\left\langle T_{x}, e^{-i(x \mid \zeta)}\right\rangle,(x \mid \zeta)=\sum_{j} x_{j} \zeta_{j},
$$

is an entire function in $\boldsymbol{c}^{\mathrm{n}}$ which satisfies, for some $A, N>0$, the estimates

$$
|\hat{T}(\zeta)|<A(1+|\zeta|)^{N} \exp (H(\operatorname{Im} \zeta)) \text {. }
$$

where $\zeta=\xi+i n, \xi, \eta \in \mathbb{R}^{n}, \operatorname{Im} \zeta=n$ and $H$ is the supporting function of the support of $T$, i.e.:

$$
H(n)=\operatorname{Max}\{(x \mid n): x \in \operatorname{suppT}\}
$$

Note that $H$ is also the supporting function of cv(suppt), the convex hull of suppt. The Fourier transform is an isomorphism between the convolution algebra $E^{\prime}\left(\mathbf{R}^{n}\right)$ and $\hat{E}^{\prime}\left(\mathbf{R}^{n}\right)$, the algebra of entire functions of exponential type and polynomial growth on the real axis.

A distribution $T$ will be called invertible (or $\hat{T}$ is slowing decreasing) if whenever $s \in E^{\prime}\left(R^{n}\right)$ and $\hat{S} / \hat{T}$ is an 
entire function, then there is a distribution $u \in E^{\prime}\left(R^{n}\right)$ such that $\hat{U}=\hat{S} / \hat{T}$, that is

$$
S=T * U
$$

and we have the identity

$$
\mathrm{H}_{\mathrm{S}}=\mathrm{H}_{\mathrm{T}}+\mathrm{H}_{\mathrm{U}}
$$

or, what amounts to the same thing

$$
\operatorname{cv}\left(\operatorname{suppu} u^{*}\right)=\operatorname{cv}(\operatorname{suppu})+\operatorname{cv}(\operatorname{suppT}),
$$

where, for two sets $A, B \subseteq R^{n}$ we have $A \pm B=\{x \pm y ; x \in A, y \in B\}$. We will need to use that $\mu_{r}$ is an invertible distribution. This will follow from the explicit formula for $\hat{\mu}_{r}$ given below and the characterization of invertible distributions: $T$ is invertible if and only if there is a positive constant a such that for all $\xi \in \mathbf{R}^{\text {n }}$

$$
\operatorname{Max}\left\{|T(\xi+n)|: n \in R^{n} \cdot|n|<a \cdot \log (2+|\xi|)\right\}>(a+|\xi|)^{-a}
$$

The Fourier transform of a radial distribution $T$ is a radial function, i.e.: if:

$$
\left\langle T, f \circ A^{-1}\right\rangle=\langle T, f\rangle
$$


for every $A \in O(n)$ then

$$
\hat{T}(\zeta)=\hat{T}(A \cdot \zeta)
$$

for every $A \in O(n), \quad \zeta \in \mathbb{a}^{n}$, and depends, for $\xi \in \mathbf{R}^{n}$, only on $|\xi|$. Hence we consider the associated even entire function $\tilde{T}$ of one variable by

$$
\tilde{T}(|\xi|)=\hat{T}(\xi) \text { and } \hat{T}(\zeta)=\tilde{T}\left(\left(\zeta_{1}^{2}+\ldots+\zeta_{n}^{2}\right)^{1 / 2}\right)
$$

Let us call $E_{0}^{\prime}\left(R^{n}\right)$, the space of radial distributions. This correspondence establishes an isomorphism between the algebras $\hat{E}_{0}^{\prime}\left(R^{n}\right)$ and $\hat{E}_{0}^{\prime}(R)$. Using this notation we have

$$
\tilde{\mu}_{r}(t)=n 2^{\frac{n-2}{2}} \Gamma\left(\frac{n}{2}\right) J_{\frac{n}{2}}(r t) /(r t)^{n / 2}
$$

$$
\tilde{\sigma}_{r}(t)=2^{\frac{n-2}{2}} \Gamma\left(\frac{n}{2}\right) J_{\frac{n-2}{2}}(r t) /(r t)^{\frac{n-2}{2}}
$$

and, more generally, if $f(x)=\phi(|x|)$ is a radial function of compact support

(11) $\hat{f}(\zeta)=\tilde{f}(t)=\frac{(2 \pi)^{n / 2}}{t^{\frac{n-2}{2}}} \int_{0}^{\infty} \phi(\rho) J_{\frac{n-2}{2}}(\rho t) \rho^{n / 2} d \rho \quad(|\zeta|=t)$

To show that $\mu_{r}$ is invertible it is now sufficient to recall the asymptotic development of the Bessel functions [19] on the positive real axis 


$$
J_{v}(t)=\sqrt{\frac{2}{\pi}} t^{-1 / 2} \cos \left(t-\frac{\pi}{4}-\frac{v \pi}{2}\right)+o\left(t^{-3 / 2}\right)
$$

It follows, for $|\xi|>1$ and some $C>0$

$$
\operatorname{Max}\left\{\left|\hat{\mu}_{r}(\xi+n)\right|: n \in \mathbf{R}^{n},|n|<\pi\right\}>c|\xi|^{\frac{n+1}{2}}
$$

which is the condition of invertibility.

From (12) we also obtain Mac-Mahon's asymptotic development of the positive zeros $\alpha_{k, v}$ of $J_{v}$

$$
\begin{array}{r}
0<\alpha_{1, \nu}<\alpha_{2, \nu}<\ldots \\
\alpha_{k, \nu}=(2 k+1) \frac{\pi}{2}+(2 v+1) \frac{\pi}{4}+o(1 / k)
\end{array}
$$

which will be used further on.

3. Series development of mean-periodic functions.

Let $\Omega$ be an open convex set in $\mathbf{R}^{\mathrm{n}}$ and $K=\operatorname{cv}(\operatorname{supp} \mu), \mu \in E^{\prime}\left(R^{n}\right)$. We say that a function $f \in C^{\infty}(\Omega-K)$ is mean-periodic with respect to $\mu$ if

$$
\mu * f(x)=\left\langle\mu_{y}, f(x-y)\right\rangle=0 \text { for all } x \in \Omega
$$

If an exponential-polynomial, that is a finite linear combination of terms of the form $x^{j} e^{i(x \mid \zeta)}$ 
$\left(x^{j}=x_{1}^{j} \ldots x_{n}^{j}, j_{k} \in N, 1<k<n\right)$, is mean-periodic with respect to $\mu$ then the frequencies $\zeta$ must satisfy $\hat{\mu}(\zeta)=0$ since

$$
\left(\mu \star e^{i(\cdot \mid \zeta)}\right)(x)=\hat{\mu}(\zeta) e^{i(x \mid \zeta)}
$$

When the zeros of $\hat{\mu}$ are simple no non constant monomials can appear. More generally if a monomial $x^{j}$ appears with non zero coefficient then

$$
\left(\frac{\partial|j|}{\partial \zeta^{j}}\right) \hat{\mu}(\zeta)=0
$$

for the corresponding frequency $\zeta$.

For $n=1$ there is a well-known series development for such functions in terms of the exponential polynomial solution of the same convolution equation (14) due to L. Schwartz [18], [15], [13]. The case of interest for us is $n>2, \mu$ invertible. In this case, a development in terms of integrals over the zero set of $\hat{H}$ has been proved when $\Omega=R^{n}[6]$. For $\Omega$ arbitrary convex set, a similar development has been proved in [4] but only for a very restrictive class of invertible distributions: In all these cases one obtains also some knowledge of the behavior of the terms involved in this development. Regretfully, the distributions " $r$ ' though invertible, do not satisfy the conditions required in [4], as it was shown (for a different reason) in [3], besides we are interested in $\Omega=B(0, R)$, therefore we cannot depend on any of the previously known 
results. We obtain here a series development but no precisions on the coefficients that appear in it, nevertheless the existence of this development is all we need later.

$$
\text { Proposition } 1 \text { - Let } \Omega \text { be an open convex subset in }
$$

$\mathbf{R}^{\mathbf{n}}(\mathrm{n}>2), \mu \in E^{\prime}\left(\mathbf{R}^{\mathrm{n}}\right)$ an invertible distribution,

$K=c v(s u p p \mu)$. Any function $f \in C^{\infty}(\Omega-K)$, mean periodic with respect to $\mu$ can be written as

$$
f(x)=\sum_{j>1} P_{j}(x)
$$

with $\mathrm{P}_{j}$ exponential-polynomials also mean-periodic with respect to $\mu$, and the series is convergent in the $c^{\infty}$-topology of $\Omega-K$. Furthermore, given a sequence $\left(s_{j}\right)_{j>1}$ of positive numbers, let $P_{0}=0$, we can chose the $P_{j}$ so that the absolute value of all frequencies in $P_{j+1}$ exceeds the largest absolute value of the frequencies in $P_{j}$ by at least $s_{j+1}$. Proof. Let us show first that, for any $s>0$, the exponential polynomials which are mean-periodic with respect to $\mu$ and whose frequencies lie outside the ball of center 0 and radius $s$ in $\boldsymbol{c}^{\mathrm{n}}$ are dense in the space $N=\left\{\mathrm{f} \in \mathrm{C}^{\infty}(\Omega-\mathrm{K}): \mu \star f=0\right.$ in $\left.\Omega\right\}$. $N$ is a closed subspace of a Frechet space and we only need to show that if $v \in E^{\prime}(\Omega-K)$ is orthogonal to the above exponentialpolynomials then $v$ is orthogonal to $N$. Hence $(\hat{v})$ is divisible by $\hat{\mu}$ at every point of $c^{n} \backslash \bar{B}(0, s)$. Since $n \geq 2$, by Hartogs' theorem, $(\hat{v}) \hat{\mu}$ is an entire function. since $\mu$ is invertible there is a distribution $T \in E^{\prime}\left(R^{n}\right)$ such that 


$$
\ddot{\nu}=\mu \star \ddot{r}
$$

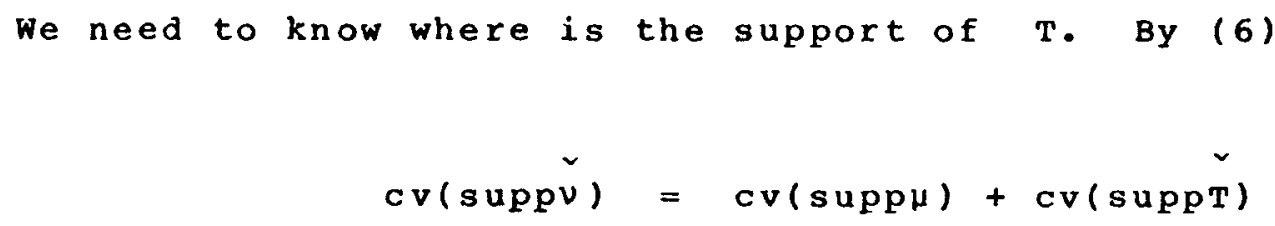

or

$$
\operatorname{cv}(\operatorname{suppt})-\mathrm{K}=\mathrm{cv}(\mathrm{supp} v) \subseteq \Omega-\mathrm{K}
$$

By the Hahn - Banach theorem one concludes that

$$
\operatorname{cv}(\operatorname{suppT}) \subseteq \Omega
$$

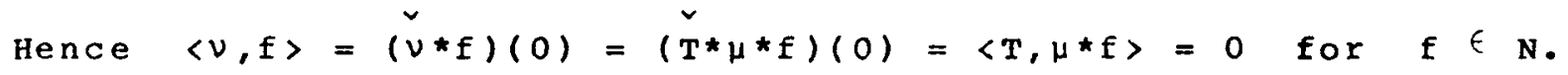
To end the proof of the proposition, we pick an exhaustion of $\Omega-K$ by convex compacts sets $K_{j}$, hence we can find $P_{1}$, exponential-polynomial with frequencies lying in $\left\{\zeta \in \mathbb{C}^{n}: \hat{\mu}(\zeta)=0,|\zeta|>s_{1}\right\}$ such that

$$
\sup _{K_{1}}|f-p,|<1
$$

Let $\sigma_{1}=$ maximum of the absolute values of frequencies in $p_{1}$. We can find $P_{2}$ with frequencies in $\left\{\zeta \in \mathbb{e}^{n}: \hat{\mu}(\zeta)=0,|\zeta|>s_{2}+\sigma_{1}\right\}$ such that

$$
\max _{|\alpha|<1} \sup _{2}\left|D^{\alpha}\left(f-P_{1}-p_{2}\right)\right|<1 / 2
$$


Continuing in this fashion we obtain the desired expansion. $\square$ Remark. One can eliminate the requirement of $\mu$ being invertible by using $[14,16.4,1]$.

From (9) we know that the zero variety of $\hat{\mu}_{r}$ is the union of the hypersurfaces

$$
\zeta^{2}=\zeta_{1}^{2}+\cdots+\zeta_{n}^{2}=\lambda_{k}^{2}
$$$$
k=1,2, \ldots
$$

where $\lambda_{k}=a_{k, n / 2} / r$. we disregard temporarily the dependence on $r$ though it will play a role later on. Furthermore the function $\tilde{\mu}_{r}(t)$ vanishes at $t=\lambda_{k}$ with multiplicity one, in fact

$$
\frac{d}{d t} \tilde{\mu}_{r}(t)=-n 2^{\frac{n-2}{2}} \Gamma\left(\frac{n}{2}\right) r J(n / 2)+1(r t) /(r t)^{n / 2}
$$

and well known properties of Bessel functions show that this expression does not vanish for $t=\lambda_{k}$. Using the asymptotic expressions (12) and (13) we obtain

(19)

$$
0 \neq \frac{d \tilde{\mu} r}{d t}\left(\lambda_{k}\right)=r n 2^{(n-1) / 2} \Gamma(n / 2)(-1)^{k+1} /\left(\lambda_{k} r\right)^{\frac{n+1}{2}}+o\left(j^{-\frac{n+3}{2}}\right)
$$

We introduce some auxiliary radial distributions $T_{r, k}$ by the formula

$$
\tilde{T}_{r, k}(t)=\frac{\tilde{\mu}_{r}(t)}{t^{2}-\lambda_{k}^{2}}
$$


They are even and entire since $\tilde{\mu}_{r}\left( \pm \lambda_{k}\right)=0$. Hence they correspond to radial distributions (in fact $c^{1,1}$ functions) whose supports are contained in the support of $\mu^{\prime}{ }^{\prime}$ i.e. $\bar{B}(0, r)$.

Furthermore they satisfy

$$
\left(\Delta+\lambda_{k}^{2}\right) T_{r, k}=-\mu_{r} \text { and }
$$

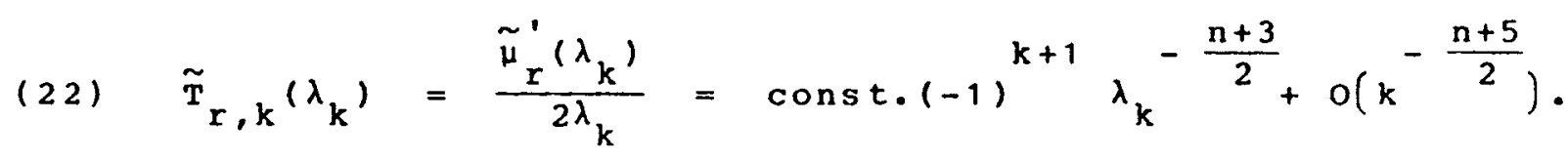

We remark that these distributions have conspicuously

appeared in previous work on the Pompeiu problem [2], [7].

$\underline{\text { Proposition } 2}$ - Let $r>0$ be fixed. For any $\rho, 0<\rho<\infty$, we can decompose $\sigma_{p}$ in the following form

$$
\sigma_{\rho}=\nu_{\rho}+\mu_{x} * s_{\rho}
$$

where $s_{\rho}$ is a radial distribution, whose support satisfies

$$
\text { supp } s_{\rho} \subseteq \bar{B}(0, \operatorname{Max}(r, \rho)-r)
$$

and $v_{\rho}$ is given explicitly by:

$$
v_{\rho}=-\sum_{k>1} \frac{\tilde{\sigma}_{\rho}\left(\lambda_{k}\right)}{\lambda_{k}^{2} \tilde{T}_{r, k}\left(\lambda_{k}\right)} \Delta T r, k
$$

hence supp $v_{\rho} \subseteq \bar{B}(0, r)$. 
Proof. We consider the series

$$
g(t)=\sum_{k \geqslant 1} \frac{\tilde{\sigma}_{\rho}\left(\lambda_{k}\right)}{\lambda_{k}^{2} \tilde{T}_{r, k}\left(\lambda_{k}\right)} t^{2} \tilde{T}_{r, k}(t)
$$

The coefficients $\lambda_{k}^{-2} \tilde{\sigma}_{\rho}\left(\lambda_{k}\right) / \tilde{T}_{r, k}\left(\lambda_{k}\right)$ are uniformly bounded by a constant depending only on $\rho$ as it can be seen from $(10)$, (13) and (22), since $\lambda_{k} \sim$ const. $k$. Therefore, if $|t|<R, \eta_{k}>2 R$ we have $\left|t^{2} \tilde{T}_{r, k}(t)\right|<$ const. $k^{-2}$ which guarantees the convergence of the series, and shows $g$ is an even entire function. We can obtain more precise estimates by picking a sequence of circles of center 0 and radii

$$
R_{j}=(4 j+n+5)^{\pi} / 4 r, j=1,2, \ldots
$$

Decomposing the sum into those terms where

$\lambda_{k}<2 R_{j}$ and $\lambda_{k}>2 R_{j}$ one can estimate the second sum over $|t|=R_{j} \quad b y$

$$
\operatorname{Max}_{|t|=R_{j}}\left|t^{2} \tilde{\mu}_{r}(t)\right| \cdot c_{o}(p)
$$

The first (finite) sum can be estimated by

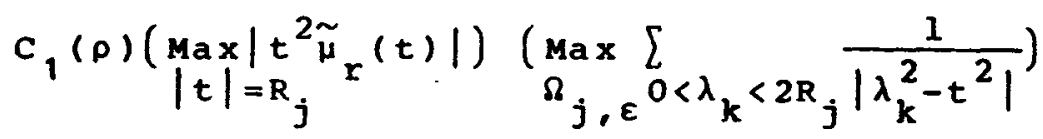

where $\Omega_{j, \varepsilon}$ is the region obtained from $|t|<R_{j}$ by remoying disks of radius $\varepsilon, 0<\varepsilon$ very small, about $\pm \lambda_{k}$ one can then see, without difficulty, that the last sum is estimated by const. $\varepsilon^{-1}$ In any case we obtain as a final estimate 


$$
\operatorname{Max}_{|t|<R_{j}}|g(t)|<c(\rho) \operatorname{Max}_{|t|<R_{j}}\left|t{ }^{2} \tilde{\mu}_{r}(t)\right| .
$$

Thus $g$ defines a radial distribution of order 2 , $v_{\rho}$, by $\tilde{v}_{\rho}=g$, one can see $v_{\rho}$ is given explicitly by (25). We also have

$$
\tilde{\sigma}_{\rho}-\tilde{v}_{\rho}=\tilde{\mu}_{r} \mathrm{~h},
$$

with $h$ even entire function since $g\left( \pm \lambda_{k}\right)=\nu_{\rho}\left( \pm \lambda_{k}\right)=\tilde{\sigma}_{\rho}\left( \pm \lambda_{k}\right)$ by (26). Since $\mu_{r}$ is an invertible distribtion it follows $h=\tilde{s}_{\rho}$ for some $s_{\rho} \in E_{o}^{\prime}\left(R^{n}\right)$. The identity (6) gives

$$
\operatorname{cv}\left(\operatorname{supp}\left(\sigma_{\rho}-v_{\rho}\right)\right)=\operatorname{cv}\left(\operatorname{supps} \rho_{\rho}\right)+\operatorname{cv}\left(\operatorname{supp} \mu_{r}\right) \cdot
$$

There are two cases to consider. If $\rho<r$, then the support on the left hand side of $(27)$ is contained in $\bar{B}(0, r)$ and cv(supp $\left.s_{p}\right)=\{0\}$, which says $s_{p}$ is a polynomial in the Laplace operator; if $\rho>r$ then the left hand side of (27) is contained in $\bar{B}(0, \rho)$, which says cv(supps $)_{\rho} \leq B(\bar{O}, \rho-x) \cdot \square$

Remark The decomposition we have just given in proposition 2 works also if we replace $\sigma_{\rho}$ by any radial distribution. We need only to change $\left(t / \lambda_{k}\right)^{2}$ by $\left(t / \lambda_{k}\right)^{2 q}$ with $q$ convenient non negative integer. In particular there is such a decomposition with $\sigma_{0}=\delta$, the Dirac mass at the origin (take $q>\frac{n+1}{4}$ ) 
Corollary 3 - Let $f$ be a $\mu_{x}$-mean-periodic function in $C^{\infty}(B(0, R))(R>r)$. Let $\left|x_{0}\right|<R-r$. Then, for any $\rho$, $0<\rho<R-\left|x_{0}\right|$ we have

$$
\lambda_{p}\left(f, x_{0}\right)=\left(v_{p} * f\right)(0)=-\sum_{k>1} \frac{\tilde{\sigma}_{p}\left(\lambda_{k}\right)}{\lambda_{k}^{2} \tilde{T}_{r, k}\left(\lambda_{k}\right)} \Delta(T r, k f)\left(x_{0}\right)
$$

Proof. It suffices to use (2) and (23).

4. Local two-circles theorem.

Let $r_{1}, r_{2}$ be two positive numbers and consider the distributions ${ }^{\mu}{ }_{r_{1}},{ }^{\mu} r_{2}$. They will have no common, meanperiodic, exponential-polynomials if and only if $\hat{\mu}_{r_{1}}$ and $\hat{\mu}_{r_{2}}$ have no common zeros. By (17) this occurs if and only if

$$
r_{1} / r_{2} \neq q u o t i e n t \text { of two zeros of } J_{n / 2}
$$

The set

$$
E_{n}=\left\{\alpha_{k, n / 2} / \alpha_{j, n / 2}: 1<j, k<\infty\right\}
$$

is the exceptional set described in the two-circles theorem.

$$
\text { Proposition } 4 \text { Let } R>r_{1}+r_{2}, r_{1} / r_{2} \& E_{n} \text {. The only }
$$
function in $C^{\infty}(B(O, R))$ which is mean-periodic with respect to both ${ }^{\mu_{r}}$ and ${ }^{\mu_{r_{2}}}$ is the zero function. Proof. We assume $r_{1}<r_{2}$. Let $f \in C^{\infty}(B(0, R))$ be $\mu_{r_{1}}$ mean periodic. By proposition 1 we have 


$$
f(x)=\sum_{j>1} P_{j}(x)(|x|<R)
$$

where the frequencies appearing in the exponential sums $\mathrm{P}_{\mathrm{j}}$ lie in

$$
\left\{\zeta \in C^{n}: \hat{\mu}_{r_{1}}(\zeta)=0\right\}=\underset{k \geqslant 1}{U}\left\{\zeta \in C^{n}: \zeta^{2}=\left(\alpha_{k, n / 2} / r_{1}\right)^{2}\right\}=\underset{k \geqslant 1}{U} v_{k} \cdot
$$

We $\mathrm{fix}$ now $k>1$, and consider $\mathrm{T}_{\mathrm{r}_{1}, k}{ }^{\star} \mathrm{f}$ which is in $C^{\infty}(B(0, R-r)$,$) , furthermore$

$$
T_{r_{1}, k} * f=\sum_{j>1} T_{r_{1}, k}{ }^{* P_{j}}
$$

If $P_{j}(x)=\sum_{\ell} c_{j, \ell} e^{i\left(x \mid \zeta_{j}, \ell\right)}$ then

$$
T_{r_{1}, k}{ }^{\star} P_{j}=\sum_{\ell} C_{j, l}{ }^{T_{r_{1}, k}}\left(\zeta_{j, l}\right) e^{i\left(x \mid \zeta_{j}, l\right)}
$$

but $\hat{T}_{r_{1}, k}\left(\zeta_{j, l}\right) \neq 0$ only if $\zeta_{j, l} \in v_{k}$ in which case we obtain the value $\tilde{\mathrm{T}}_{r_{1}, k}\left(\lambda_{k}\right)=0$ (where $\lambda_{k}$ is computed with respect to $\left.r_{1}\right)$. Therefore

$$
T_{r_{1}, k}{ }^{f}=\tilde{T}_{r_{1}, k}\left(\lambda_{k}\right) \sum_{j>1} P_{j, k}
$$

where $P_{j, k}$ is the sum of the terms in $P_{j}$ whose frequencies lie in $v_{k}$. This series is convergent in $C^{\infty}\left(B\left(0, R-r_{1}\right)\right)$. We convolve now with ${ }_{r_{2}}$. We obtain

$$
\left.{ }_{r_{2}}{ }^{\star\left(T_{r_{1}, k}\right.}{ }^{* f}\right)=\tilde{T}_{r_{1}, k}\left(\lambda_{k}\right) \tilde{\mu}_{r_{2}}\left(\lambda_{k}\right) \sum_{j>1} P_{j, k}
$$


since ${ }^{\mu}{ }_{2}$ is also a radial distribution. The expansion (31) is valid in $C^{\infty}\left(B\left(0, R-r_{1}-r_{2}\right)\right)$. Since $f$ is also ${ }^{\mu} r_{2}$ - mean periodic we have

$$
0=\left(T_{r_{1}, k}{ }^{\star \mu} r_{2}{ }^{\star f}\right)(x)=\tilde{\mu}_{r_{2}}\left(\lambda_{k}\right)\left(T_{r_{1}, k} * f\right)(x)
$$

for $|x|<R-r_{1}-r_{2}$. The hypothesis $r_{1} / r_{2} \notin E_{n}$ now implies that $\tilde{\mu}_{r_{2}}\left(\lambda_{k}\right) \neq 0$. Hence

$$
\left(T_{r_{1}, k} * f\right)(x)=0 \text { for }|x|<R-r_{1}-r_{2}
$$

On the other hand we have (by (22))

$$
\left(\Delta+\lambda_{k}^{2}\right)\left(T_{r_{1}, k} * f\right)=-\left(f * \mu_{r_{1}}\right)=0 \text { in }|x|<R-r_{1}
$$

hence $T_{r_{1}, k^{*}}$ is a real analytic function in $|x|<R-r_{1}$. We conclude that

$$
\left(T_{r_{1}, k^{*}}\right)(x)=0 \text { for }|x|<R-r_{1}
$$

Applying now corollary 3, formula (28), we have

(34)

$$
\lambda_{p}(f, x)=0 \text { whenever }|x|<R-r_{1}, 0<\rho<R-|x|
$$

(we are allowed to take $\rho=0$ by continuity). In particular 


$$
f(x)=0 \text { for }|x|<R-r_{1}
$$

To finish the proof of the proposition we need to show $f$ is zero in the remaining annulus, we do that using (34). It is at this point that we use cormack-Quinto [12]. For any $y$ $B(0, R)$, consider $R(f)(y)=\lambda|y| / 2(f, y / 2)$. This is the Radon transform on spheres through the origin discussed in [12]. We want to show $\operatorname{Rf}(y)=0$. We only need to verify that the conditions stated in (34) are valid. Here $\rho=|y| / 2, x=y / 2$, hence

$$
R-|x|=R-\frac{|y|}{2}=R-\rho>R / 2>\rho
$$

The only condition left to see is that $|x|<R-r_{1}$. We have $2 r_{1}<r_{1}+r_{2}<R$ hence $r_{1}<R / 2$ and $R-r_{1}>R / 2$, therefore $|x|<R-r_{1}$ holds.

By [12, corollary 2] $f(y)=0$. (we note that in [12], they require that $f C^{\infty}\left(R^{n}\right)$ while we only have $f \in C^{\infty}(B(0, R))$ but the proof of corollary 2 depends on an explicit inversion formula for the Radon transform on spheres which uses, for each y, values of $f$ in a neighborhood of $\bar{B}(0,|y|)$, )

Remark. The crucial point of the proof above is (32). One does not really need the whole strength of propositon 1 to obtain it. One can get by using the density of the exponential polynomial solutions in the sub-space $N$ introduced in propositon 1. Nevertheless, we feel that the proof is clearer using the expansion (16) as we have done. 
We want to show that the condition $R>r_{1}+r_{2}$ is sharp. It is easier to show this under the slight restriction that $r_{2} / r_{1}$ is not too well approximated by elements in $E_{n}$. Definition. - For $N>0$, we say that a positive number is N-well approximated by points in $E_{n}$ if, for every $\ell \geq 1$, there are indices $j, k$ such that

$$
\left|r-\alpha_{k} / \alpha_{j}\right|<\frac{1}{\ell j}
$$

where $\alpha_{k}=\alpha_{k, n / 2}$

Proposition 5 For any $N>2$, the set of numbers $N$-well approximates by $E_{n}$ has zero measure in $(0, \infty)$

Proof. Given $p, q, 0<p<r<q$ and $\nu>0$, from (13)

we have

$$
\alpha_{k, v}=\alpha_{k}=(2 k+1) \pi / 2+(2 v+1) \pi / 4+0(1 / k)
$$

Therefore, if $r$ satisfies (35), for $\ell>1$, we have

$$
|r \cdot j-k+A r+B|<C
$$

for some constants $A, B, C$. Hence

$$
\mathrm{pj}-\mathrm{C}_{1}<\mathrm{k}<\mathrm{q} \mathrm{j}+\mathrm{c}_{2}
$$

for some constant $c_{1}, c_{2}>0$. Hence the cardinal of the set of $k$ satisfying $(36)$ is bounded by $(q-p) j+L$, L constant $>0$. 
Now, the set of $N$-well approximated numbers in $[p, q]$ is

(37)

$$
\bigcap_{\ell>1} \underset{j, k>1}{U}\left\{r: p<r<q,\left|r-\alpha_{k} / \alpha_{j}\right|<1 / \ell j^{N}\right\}
$$

For $\ell$ fixed, the Lebesgue measure

$\left|\bigcup_{j, k}\left\{r: p<r<q,\left|r-\alpha_{k} / \alpha_{j}\right|<1 / j^{N}\right\}\right|<\frac{2}{l} \sum_{j>1} \frac{(q-p) j+L}{j}<\frac{C_{3}}{\ell}$

$\left(c_{3}>0\right)$ since $N>2$. Therefore the set (37) has zero measure and by letting $q=p+1, p \in N$ we obtain the proposition.

It is interesting to compare proposition 5 with [8, Lemma

2.1] where examples of numbers which are not 2-well approximated

by $E_{n}(n=2)$ are discussed. It might be that these include all

rationals $\neq 1$, all quadratic irrationals $\neq 1$, but no such theorem seems to be known. Also, it is easy to see that, for $N<1$, every positive number is $N$-well approximated by $E_{n}$. Proposition 6. - Let $r_{1}, r_{2}$ be two positive numbers such that $\mathrm{r}_{2} / \mathrm{r}_{1}$ is not $\mathrm{N}$-well approximated by $\mathrm{E}_{\mathrm{n}}$. Denote by $\lambda_{k}$ the positive zeros of $\tilde{\mu}_{r_{1}}$. There is a positive constant c such that

$$
\left|\tilde{\mu}_{r_{2}}\left(\lambda_{k}\right)\right|>\frac{C}{k_{k}+\frac{n-1}{2}}
$$

Proof. Let us denote $\alpha_{k}=\alpha_{k, n / 2}$ Recall that $\lambda_{k}=\alpha_{k} / r_{1}$ and that

$$
\tilde{u}_{r_{2}}(t)=\text { const. } \frac{J_{n / 2}\left(r_{2} t\right)}{\left(r_{2} t\right)^{n / 2}}
$$


From the asymptotic development (13) we have

$$
\alpha_{k+1}-\alpha_{k}=\pi+o(1 / k)
$$

Hence, if $k$ is fixed and $j_{k}$ is chosen such that $\left|r_{2} \lambda_{k}-\alpha_{j}\right|$ is minimal we have

$$
\varepsilon_{k}=\left|r_{2} \lambda_{k}-\alpha_{j k}\right|<\pi / 2+o(1 / k)
$$

Let us distinguish two cases: $\varepsilon_{k}<\pi / 4$ or not. In the second case we have

$$
\begin{gathered}
\left|\cos \left(r_{2} \lambda_{k}-\frac{(n+1)}{4} \pi\right)\right|=\left|\cos \left( \pm \varepsilon_{k}+\left(2 j_{k}+1\right) \frac{\pi}{2}+o\left(\frac{1}{k}\right)\right)\right| \\
=\left|\sin \left(\varepsilon_{k}+o(1 / k)\right)\right|>\frac{\sqrt{2}}{2}+o\left(\frac{1}{k}\right)>c_{0}>0
\end{gathered}
$$

for large $k$. In this case the asymptotic development (12) gives the estimate

$$
\left|\tilde{\mu}_{r_{2}}\left(\lambda_{k}\right)\right|>c_{1} k^{-\frac{n+1}{2}}
$$

for some $c_{1}>0$ and all large $k$.

By hypothesis we have that for all $j, k$

$$
\left|\frac{r_{2}}{r_{1}}-\frac{a_{1}}{\alpha_{k}}\right|>\frac{c_{2}}{k^{N}}\left(c_{2}>0\right)
$$


Therefore $\varepsilon_{k}>c_{3} / k^{N-1}\left(c_{3}>0\right)$. Suppose also $\varepsilon_{k}<\pi / 4$. By the mean-value theorem there is a $\xi$ between $\alpha_{j_{k}}$ and $r_{2} \lambda_{k}$ such that

$$
\frac{J_{n / 2}\left(r_{2} \lambda_{k}\right)}{\left(r_{2} \lambda_{k}\right)^{n / 2}}=-r_{2} \frac{J_{n / 2+1}(\xi)}{\xi^{n / 2}} \cdot\left(r_{2} \lambda_{k}-\alpha_{j k}\right)
$$

$\left(\operatorname{Recall} \mathrm{J}_{\mathrm{n} / 2}\left(\alpha_{j_{k}}\right)=0.\right)$ Note that $\delta_{k}=\left|\xi-\alpha_{j}\right|<\varepsilon_{k}<\pi / 4$. Again by (12) we have to estimate

$$
\begin{aligned}
\cos \left(\xi-\frac{\pi}{4}-\frac{(n+2) \pi}{4}\right) & =\cos \left( \pm \delta_{k}+\left(2 j_{k}+1\right) \frac{\pi}{2}+\frac{(n+1) \pi}{4}-\frac{(n+2) \pi}{4}+o(1 / k)\right) \\
& =\cos \left( \pm \delta_{k}+j_{k} \pi+o(1 / k)\right) \\
& = \pm \cos \delta_{k}+o(1 / k) .
\end{aligned}
$$

Then

$$
\left|\tilde{\mu}_{r_{2}}\left(\lambda_{k}\right)\right|>\frac{C_{4}}{k_{k}^{N-1+\frac{n+1}{2}}}\left(c_{4}>0\right) .
$$

Since $N>1$ the estimate (38) holds in both cases.

proposition 7 let $f$ be a function in

$L_{1 O C}^{1}(B(0, R)), g \in L_{1 O C}^{1}(B(O, R))$, suppg $\subseteq B(0, r), g$ radial. For $\left|x_{0}\right|<R-r$ and $\rho<R-r-\left|x_{0}\right|$ we have

(40)

$$
\lambda_{p}\left(f * g, x_{0}\right)=\left(\lambda|\cdot|\left(f, x_{0}\right) * g(\cdot)\right)(y) \quad(|y|=p)
$$


(The notation indicates that we are convolving in the variable denoted by a dot).

Proof. Recall that the average $\lambda_{\rho}\left(f, x_{0}\right)$ can also be computed by

$$
\lambda_{p}\left(f, x_{0}\right)=\int_{O(n)} f\left(x_{0}+A y\right) d A
$$

where $y$ is any point with $|y|=\rho, o(n)$ is the orthogonal group and $d A$ is the normalized Haar measure. Let

$$
\phi(y)=\left(\lambda|\cdot|^{\left.\left(f, x_{0}\right) * g(\cdot)\right)(y)}\right.
$$

we have

$$
\begin{aligned}
& \phi(y)=\int_{R^{n}}\left(\int_{O(n)} f\left(x_{0}+A(y-x) d A\right) g(x) d x\right. \\
& =\int_{O(n)}\left(\int_{R} f\left(x_{0}+A(y-x)\right) g(x) d x\right) d A \\
& \text { set } u=A x \text { then } g(x)=g(u) \text { and } d x=d u \text { Hence } \\
& \phi(y)=\int_{O(n)}\left(\int_{R^{n}} f\left(x_{0}+A y-u\right) g(u) d u\right) d A \\
& =\lambda_{\rho}\left(f * g, x_{0}\right)
\end{aligned}
$$


(41)

$$
\left(g(x) * \frac{\frac{J_{n-2}}{2}(\alpha|x|)}{(a|x|)^{\frac{n-2}{2}}}\right)(y)=\tilde{g}(\alpha) \frac{\frac{J_{n-2}}{2}(\alpha|y|)}{(\alpha|y|)^{\frac{n-2}{2}}}
$$

Proof. Let $\xi \in \mathbf{R}^{\mathbf{n}}$ be any vector with $|\xi|=\alpha$, then

$$
\begin{gathered}
\left(g(\cdot) * e^{i(\xi \mid \cdot)}\right)(y)=\hat{g}(\xi) e^{i(\xi \mid y)} \\
=\tilde{g}(\alpha) e^{i(\xi \mid y) .}
\end{gathered}
$$

On the other hand

$$
\begin{gathered}
\lambda_{\rho}\left(e^{i(\xi \mid \cdot)}, 0\right)=\tilde{\sigma}_{\rho}(\alpha) \\
=22^{\frac{n-2}{2}} r(n / 2) \frac{\frac{J_{n-2}}{2}(\alpha \rho)}{(\alpha \rho)^{\frac{n-2}{2}}} .
\end{gathered}
$$

Applying now to (42) proposition 7 we obtain the desired formula (41).

Proposition 9. Let $r_{1}, r_{2}$ be two positive numbers such that $r_{2} / r_{1}$ is not $N$-well approximated by $E_{n}$. Let $R$ be any number, $\max \left(r_{1}, r_{2}\right)<R<r_{1}+r_{2}$. Then there is a non zero radial function $f \in C^{\infty}(B(0, R))$ which is mean periodic with respect to ${ }^{\mu}{ }_{1}$ and ${ }^{\mu} r_{2} \cdot$

Proof. Let $\left.\phi \in D(] 0, r_{1} l\right), \phi \neq 0$ such that $\operatorname{supp} \phi \underline{C}\left[R-r_{2}, r_{1} l\right.$ It follows from [16, theorem 2.1 page 247] that $\phi$ admits a geries development of the form 
(43)

$$
\phi(t)=\sum_{k>1} a_{k} \frac{\frac{J_{n-2}}{2}\left(\lambda_{k} t\right)}{\left(\lambda_{k} t\right)^{\frac{n-2}{2}}}
$$

where $\lambda_{k}=\alpha_{k, n / 2} / r_{1}$. This is the sturm-Liouville expansion for a boundary value problem singular at $t=0$ and derivative equal to zero at $t=r_{1}$. It can be seen by successive integrations of parts that

$$
\left|a_{k}\right|=o\left(k^{-p}\right) \text { for every } p>0
$$

since $x_{2} / x_{1}$ is not $N$-well approximated by $E_{n}$ we see that

$$
b_{k}=a_{k} / \tilde{\mu}_{r_{2}}\left(\lambda_{k}\right)
$$

satisfies the same estimates as $a_{k}$ (Proposition 6) Hence the function

$$
f(x)=\sum_{k>1} b_{k} \frac{\frac{J_{n-2}}{2}\left(\lambda_{k}|x|\right)}{\left(\lambda_{k}|x|\right)^{\frac{n-2}{2}}}
$$

is a $c^{\infty}$ radial function in $R^{n}$, f 8, it follows that $f$ is $\mu_{r_{1}}$ mean-periodic. Furthermore

$$
\begin{aligned}
\left(\mu_{r_{2}}{ }^{* f}\right)(x) & =\sum_{k>1} b_{k} \tilde{\mu}_{r_{2}}\left(\lambda_{k}\right) \frac{\frac{J_{n-2}}{2}\left(\lambda_{k}|x|\right)}{\left(\lambda_{k}|x|\right)^{\frac{n-2}{2}}} \\
& =\phi(|x|)
\end{aligned}
$$


which is zero in $B\left(0, R-r_{2}\right)$ and therefore the function $f$ restricted to $B(0, R)$ is $\mu_{r_{1}}$ and $\mu_{r_{2}}$ mean-periodic. $\square$

The above propositions can be sumarized by the following: Theorem 10. Let $r_{1}>0$ and $r_{2}>0$ be such that $r_{2} / r_{1} \in E_{n}$. The necessary and sufficient condition on a open set $\Omega$ of $\mathbf{R}^{\mathrm{n}}$ so that the only distribution $\mathrm{T} \in D^{\prime}(\Omega)$ which can be mean-periodic with respect to both $\mu_{r_{1}}$ and ${ }^{\mu_{r_{2}}}$ is $T=0$, is that $\Omega$ is the reunion of balls of radii strictly larger than $r_{1}+r_{2}$.

An amusing corollary of theorem 10 is the following: Corollary 11 If $r_{2} / r_{1} \in E_{2}, r_{1}+r_{2}<R$ and $f \in C(B(O, R))$ then the conditions

$$
\int_{\partial B\left(z, r_{j}\right)} f(\zeta) d \zeta=0 \text { for every } z,|z|<R-r_{j}(j=1,2),
$$

imply that $f$ is holomorphic in the disk $B(0, R)$.

\section{Generalizations}

After a first version of this paper was written, Professor zalcman pointed out to us that J. D. Smith [17] had also proved the two-circles, starting precisely with a local version of it. It turns out it was not as sharp as proposition 4 since he required $R>2 r_{1}+r_{2}$. Furthermore, Dr. Smith had also indicated to professor zalcman. that his method did not generalize to the other problems discussed in [20], e.g. the converse of the mean value property for harmonic functions. The aim of this section is to show that the methods used above do generalize. 
Definition. We say that a radial distribution $\mu$ of compact support is hyperbolic if:

(i) $\mu$ is invertible, and

(ii) there is a constant $C$ such that every zero $\lambda$ of $\tilde{\mu}$ satisfies

$$
|\operatorname{Im} \lambda| \leq c \log (2+|\lambda|)
$$

Theorem 12 Let $\mu_{1}, \mu_{2}, \ldots$ be a (possibly infinite) family of radial distributions of compact support, $\operatorname{cv}\left(\operatorname{supp} \mu_{j}\right)=\bar{B}\left(0, r_{j}\right)$. suppose $\left\{z \in C^{n}: \mu_{j}(z)=0 \forall j\right\}=\emptyset, \mu_{1}$ is hyperbolic, and $R-I_{1}>\sup I_{j}$. Then $\left\{f \in D\left(B(0, R): \mu_{j} \star f=0 \forall j\right\}=\{0\}\right.$. Proof: Due to the condition on $R$ we can assume $f \in C^{\infty}(B(0, R))$ as done before. The proof that leads to (32) can be repeated almost verbatim just using for each $\lambda_{k}$, zero of $\tilde{\mu}_{1}$, a convenient $\mu_{j}(j>2)$ with $\tilde{\mu}_{j}\left(\lambda_{k}\right) \neq 0$. We obtain

$$
T_{k, s} * f(x)=0 \text { for }|x| R-r_{1}-\sup _{j} r_{j}
$$

where $\tilde{T}_{k, s}(t)=\tilde{\mu}_{1}(t)\left(t^{2}-\lambda_{k}^{2}\right)^{-s}, 1<s<m_{k}, m_{k}=$ multiplicity of $\lambda_{k}$ as a root of $\tilde{\mu}_{1}$. On the other hand

$$
(-1)^{s}\left(\Delta+\lambda_{k}^{2}\right)^{s}\left(T_{k, s} * f\right)=\mu_{1} * f=0 \text { in } B\left(0, R-r_{1}\right) \text {, }
$$

therefore, $T_{k, s} \star f$ is real analytic, and hence 


$$
T_{k, 5} * f=0 \text { in } B\left(0, R-r_{1}\right) \text {. }
$$

as before. It is at this point we have to be more careful to prove the correct version of proposition 2. It will be replaced by the following:

$\underbrace{\text { Lemma } 13}_{\infty}$ Let $\Lambda=\left\{\lambda_{k}\right\}=$ set of distinct zeros of $\tilde{\mu}_{1}$, then $\Lambda=\underset{j=0}{U} \Lambda_{j}$, where the $\Lambda_{j}$ are finite and mutually disjoint sets. There is also a positive integer $q$ such that for any $\rho, 0<\rho<\infty$ we can write

$$
\sigma_{\rho}=v_{\rho}+\mu_{1} * s_{\rho},
$$

where $\nu_{p}$ ' $s_{p}$ are radial distributions satisfying

$$
\operatorname{supp} v_{\rho} \underline{C} \bar{B}\left(0, r_{1}\right)
$$

$$
\text { supp } s_{\rho} \subseteq \bar{B}\left(0, \operatorname{Max}\left(r_{1}, \rho\right)-r_{1}\right)
$$

Furthermore,

$$
v_{p}=\sum_{j=0}^{\infty} \Delta \Delta^{q_{p}}, j^{\prime}
$$

a convergent series in $E_{0}^{\prime}\left(R^{n}\right)$, each $v_{p, j}$ a finite linear combination of the distributions $T_{k, j}, \lambda_{k} \in \Lambda_{j}, 1<s<m_{k}$ (if $m_{0}>1$ then one denotes by $\Delta^{q_{\nu}}{ }_{p, 0}$ not only a finite linear combination of $\Delta^{q} T_{k, s}, \lambda_{k} \in \Lambda_{0}$ but also of $T_{0, m_{0}}, \Delta T_{0, m_{0}}, \ldots, \Delta^{q-1} T_{0, m_{0}}, l$ 
Once this lemma has been proved, the proof of Theorem 12 is achieved the same way as it was done in proposition 4 and we note that the hypotheses imply $2 r_{1}<R$. Proof of Lemma 13 The proof of this lemma proceeds as in proposition 2 by interpolating the values of $\tilde{\sigma}_{\rho}$ on the variety of zeros of $\tilde{\mu}_{1}$ (counted with multiplicities). We have to repeat with due care the procedure used in [13], [15], [18] since we need the precise statement (51), (52), and (53).

First we note that as in [5, lemma 4] (cf. also [11, p. 50]), the condition of hyperbolicity and the minimum modulus theorem allow us to construct a family of a Jordan quadrilaterals $\Gamma_{k}, k \in \mathbf{z}$ symmetric with respect to the real axis and enjoying the following properties:

(54) for some $d>0$ the horizontal sides lie on the curves

$$
\operatorname{Im} z= \pm \log (d+|\operatorname{Rez}|)
$$

and the vertical sides are arcs of circles.

(55) $0 \in$ int $\Gamma_{0}$ which is symmetric with respect to the origin (i.e. if $z \in \Gamma_{0}$ then $-z \in \Gamma_{0}$ also).

(56) for $k \neq 0, \Gamma_{-k}$ is the symmetric of $\Gamma_{k}$ with respect to the origin. 
(57) for $j \neq k, \overline{\text { int } \Gamma_{g}} \cap{\overline{\text { int } \Gamma_{k}}}_{0}=\emptyset$, furthermore, for some positive number a we have that if $z \in \Gamma_{j}$ ' $\operatorname{dist}\left(z, \Gamma_{k}\right)>(a+|z|)^{-a}$ for any $k \neq j$.

(58) for some positive constant b we have:

$$
\text { diam } \Gamma_{j}<b(1+|z|)^{b}
$$

and

$$
\text { length } \Gamma_{j}<b(1+|z|)^{b} \text {, }
$$

for any $z \in \overline{\overline{i n t}_{j}}$, any $j$.

(59) there is a constant $c>0$ such that for any $j$, and any $z \in \Gamma_{j}$ we have

$$
\left|\tilde{\mu}_{1}(z)\right|>(c+|z|)^{-c}
$$

and this inequality is valid even for those $z$ such that $\operatorname{dist}\left(z, \Gamma_{j}\right)<1 / 2(a+|z|)^{-a}$ (the same a as in (57))

$$
\Lambda \subseteq \bigcup_{j=-\infty}^{\infty}\left(\operatorname{int} \Gamma_{j}\right) .
$$

$$
\text { for some } d>0 \text { : if } j>1, z \in r_{j} \text {, then }|z|>j / d \text {. }
$$

$$
\Lambda_{0}=\Lambda \text { int } \Gamma_{0}, \Lambda_{j}=\Lambda \quad\left(\text { int } \Gamma_{j} \cap \text { int } \Gamma_{-j}\right) . j>1
$$


For the sake of definiteness we will index the points in $\Lambda$ so that $\lambda_{0}=0$, and, for $k>1$, either $\operatorname{Re} \lambda_{k}>0$ or $\operatorname{Re} \lambda_{k}=0$ and Im $\lambda_{k}>0$, and, finally, $\lambda_{-k}=-\lambda_{k}$. Now consider the even entire function

$$
f(t)=t^{2 q} \tilde{\mu}_{1}(t)
$$

for $q$ a positive integer to be chosen conveniently later on. we note that if $t \notin{\overline{\text { int } \Gamma_{j}}}_{j} U{\overline{\text { int } \Gamma_{j}}}_{j}$ then

$$
\phi_{j}(t)=\frac{1}{2 \pi i} \int_{\Gamma j} \frac{\tilde{\sigma}_{\rho}(s)}{f(s)} \frac{d s}{s-t}+\frac{1}{2 \pi i} \int_{\Gamma_{-j}} \frac{\tilde{\sigma}_{\rho}(s)}{f(s)} \frac{d s}{s-t}
$$

(where we disregard the second term if $j=0$ ) is an even function which is a linear combination of terms of the form $\left(t^{2}-\lambda_{k}^{2}\right)^{-s}$, for $\lambda_{k} \in \Lambda_{j}$ and $1<s<m_{k}$ if $k>1$, $1<s<m_{0}+2 q$ if $k=0$. Hence $\phi_{j}$ can be defined as a rational function throughout $c$ and the function $f(t) \phi_{j}(t)$ is an even entire function. We want to show now that $q$ can be chosen so that

$$
g(t)=\sum_{j=0}^{\infty} f(t) \phi_{j}(t)
$$

is in $\tilde{E}_{0}^{\prime}\left(R^{n}\right)$ and the series converges in the topology of $\tilde{E}_{0}^{\prime}\left(R^{n}\right)$.

In fact, we have that for $|\operatorname{Im} t|<\log (d+|\operatorname{Re} t|)$ there is some $N>0$ such that 
$(66)$

$$
\left|\tilde{\sigma}_{\rho}(t)\right|<C(\rho)(1+|t|)^{N}
$$

and also

$$
\left|\tilde{\mu}_{1}(t)\right|<c_{0}(1+|t|)^{N} \text {. }
$$

Therefore, for some $N_{1}>0$ sufficiently large, if

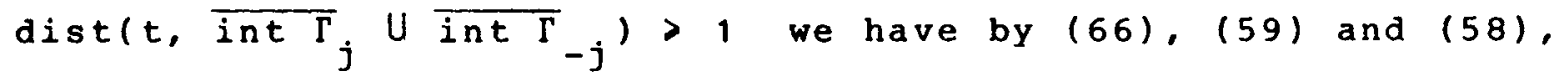
that with respect to an arbitrary point $z \in{\overline{\text { int } \Gamma_{j}}}$ which we can take it to be the point in the positive real axis closest to the origin,

$$
\left|\phi_{j}(t)\right|<\left(N_{1}+|z|\right)^{N}|z|^{-2 q}<\text { const. } j^{-2}
$$

by (61) (just take $2 q>N_{1}+2$ ). Therefore, under the same condition on $t$ we have

$$
\left|f(t) \phi_{j}(t)\right|<c_{1} j^{-2}(1+|t|)^{M} e^{r_{1}|I m t|}
$$

Using the condition (58) on the diameter of $r_{j}$ and (67), this estimate remains valid throughout $\mathfrak{c}$, after possibly increasing $C_{1}, M$. This shows that the $\nu_{\rho} \in E_{0}^{\prime}\left(R^{n}\right)$ defined by

$$
\tilde{v}_{\rho}(t)=g(t)
$$

satisfies (51). It is also clear that the distributions $v_{\rho, j}$ such that $t^{2 q} v_{\rho, j}=f(t) \phi_{j}(t)$ have the properties required 
[16] B.M. Levitan and I.S. Sargsjan, Introduction to spectral theory, Translations of Math. Monograph AMS 39(1975).

[17] J.D. Smith, Harmonic analysis of scalar and vector fields in $R^{n}$, Proc. Camb. Phil. Soc. 72(1973), 403-416.

[18] L. Schwartz, Théorie générale des fonctions moyennepériodiques, Ann. of Math. 48(1947) 857-929.

[19] G. Watson, Theory of Bessel Functions, Cambridge Univ. Press, London/New York (1966).

[20] L. Zalcman, Analyticity and the Pompeiu Problem, Arch Rat. Mech. Anal 47(1972), 237-254.

[21] L. Zalcman, offbeat integral geometry, Amer. Math. Monthly $87(1980), 161-175$. 
defined by

$$
\tilde{\mu}_{j}(t)=t^{-2}{\widetilde{\sigma_{r}^{-\delta}}}_{r_{j}}(t)=\left(\tilde{\sigma}_{r_{j}}(t)-1\right) / t^{2}
$$

are hyperbolic. The hypothesis on $r_{1} / r_{2}$ guarantees these two entire functions have no common zeros. Theorem 13 shows now that the distribution $\Delta u$ is zero in $B(O, R)$.

Remark As mentioned in [20], Delsarte proved this theorem in $R^{n}$. He also showed that $H_{n}$ is finite and $H_{3}=\{1\}$. Hence, at least for dimension 3 , any pair of distinct positive value $r_{1}, r_{2}$ would work in the above corollary.

The several other results in [20] can now be carried over to the local case without difficulty. It remains as an open question for the moment the elimination of the invertibility condition on $\mu_{1}$, which could probably be done following the Euclidean summation method of [6]. More interesting, in our view, is to try to extend this theorem to non-compact symmetric spaces of rank 1 or even to the Euclidean group thus obtaining a local version of the Pompeiu problem considered in [9].

As an example of this let us mention the following corollary of Theorem 13 .

Corollary 15 Let $R>\sqrt{n} a$, if $f \in L_{10 c}^{1}(B(0, R))$ has zero integral over any $n$-cube of side a contained in $B(O, R)$, then $f=0$ a.e.

Proof Following the ideas from [9] we see we can consider all radial distributions $\mu$ whose Fourier tranforms are of the form 
(69)

$$
\int_{O(n)} \hat{x}_{Q}(k \zeta) \hat{T}(k \zeta) d x=\hat{\mu}(\zeta)
$$

where $Q$ is the cube $[-a / 2, a / 2]^{n}$ and $T$ is a distribution of compact support in the ball $B(0, \varepsilon), \varepsilon+\sqrt{n} a<R$. Then, for any such $\mu, \operatorname{cv}(\operatorname{supp} \mu) \quad \dot{B}(0, r)$, and $f$ will satisfy the equations :

$$
\mu \star f=0 \text { in } B(0, R-r)
$$

Bince this set of distributions generates the same closed ideal in $E^{\prime}\left(R^{n}\right)$ as those are considered in $[9, p .602]$, then their Fourier tranforms have no common zeros [9, section 9]. It only remains to find a distribution that plays the role of $\mu_{1}$ in Theorem 13. The easiest one is obtained when

$$
T=\frac{\partial^{2 n} x_{Q}}{\partial x_{1}^{2} \cdots \partial x_{n}^{2}}
$$

An easy computation shows that in this case, for $\mu_{1}=$ average over $O(\mu)$ of $\frac{\partial^{2 n}}{\partial x_{1}^{2} \ldots \partial x_{n}^{2}} x_{Q^{\prime}}$ we have

$$
\tilde{\mu}_{1}(t)=\text { const. } t^{(n / 2)+1} J_{\frac{3 n-2}{2}}(\sqrt{n} \text { at/2) }
$$

which is clearly hyperbolic. (For $n=2$, this can be obtained from sonine second finite integral $[19$, p.376].) 